\title{
The $\left[70,1^{-}\right]$baryon multiplet in the $1 / N_{c}$ expansion revisited
}

\author{
N. Matagne and Fl. Stancu \\ University of Liège, Physics Department, \\ Institute of Physics, B5, \\ Sart Tilman, B-4000 Liège 1, Belgium \\ E-mail: nmatagne@ulg.ac.be, fstancu@ulg.ac.be
}

\begin{abstract}
.
The mass splittings of the baryons belonging to the $\left[\mathbf{7 0}, 1^{-}\right]$-plet are derived by using a simple group theoretical approach to the matrix elements of the mass formula. The basic conclusion is that the first order correction to the baryon masses is of order $1 / N_{c}$ instead of order $N_{c}^{0}$, as previously found. The conceptual difference between the ground state and the excited states is therefore removed.
\end{abstract}

Keywords: baryon spectroscopy, large $N_{c}$ QCD

PACS: 12.39.-x,11.15.Pg,11.30.Hv

Introduction. The $1 / N_{c}$ expansion of $\mathrm{QCD}$ [1] is a powerful theoretical tool which allows to systematically analyze baryon properties. The success of the method stems from the discovery that the ground state baryons have an exact contracted SU $\left(2 N_{f}\right)$ symmetry when $N_{c} \rightarrow \infty[2,3], N_{f}$ being the number of flavors. A considerable amount of work has been devoted to the ground state baryons, summarized in several review papers as, e. g., $[4,5]$. For $N_{c} \rightarrow \infty$ the ground state baryons are degenerate. For large $N_{c}$ the mass splitting starts at order $1 / N_{c}$. The applicability of the approach to excited states is a subject of current investigation. The experimental facts indicate a small breaking of $\mathrm{SU}\left(2 N_{f}\right)$ which make the $1 / N_{c}$ studies of excited states plausible. When the $\mathrm{SU}\left(N_{f}\right)$ symmetry is exact, the baryon mass operator is a linear combination of terms

$$
M=\sum_{i} c_{i} O_{i},
$$

with the operators $O_{i}$ having the general form

$$
O_{i}=\frac{1}{N_{c}^{n-1}} O_{\ell}^{(k)} \cdot O_{S F}^{(k)}
$$

where $O_{\ell}^{(k)}$ is a $k$-rank tensor in $\mathrm{SO}(3)$ and $O_{S F}^{(k)}$ a $k$-rank tensor in $\mathrm{SU}(2)$, but invariant in $\mathrm{SU}\left(N_{f}\right)$. The latter is expressed in terms of $\mathrm{SU}\left(N_{f}\right)$ generators. For the ground state one has $k=0$. The first factor gives the order $\mathcal{O}\left(1 / N_{c}\right)$ of the operator in the series expansion. The lower index $i$ represents a specific combination of generators, see Table 1. In Eq. (1), each $O_{i}$ is multiplied by an unknown coefficient $c_{i}$ which is a reduced matrix element. All these coefficients encode the QCD dynamics 
and are obtained from a fit to the existing data. Additional terms are needed if $\mathrm{SU}\left(N_{f}\right)$ is broken.

Excited states. The excited states can be grouped into excitation bands with $\mathrm{N}$ $=1,2,3$, etc. units of excitation energy. Among these, the states belonging to the $\mathrm{N}=1$ band, described by the $\left[\mathbf{7 0}, 1^{-}\right]$-plet, have been most extensively studied, either for $N_{f}=2$, see e.g. $[6,7,8]$ or for $N_{f}=3[9]$. The conclusion was that the splitting starts at order $N_{c}^{0}$.

The method has been applied to the $\mathrm{N}=2$ band multiplets $\left[\mathbf{5 6}^{\prime}, 0^{+}\right]$for $N_{f}=2$ [10], $\left[\mathbf{5 6}, 2^{+}\right]$for $N_{f}=3[11]$ and $\left[\mathbf{7 0}, \ell^{+}\right]$for $N_{f}=2[12]$ and $N_{f}=3[13]$ and also to $N_{f}=3$ baryons [14] of the $\left[\mathbf{5 6}, 4^{+}\right]$-plet ( $\mathrm{N}=4$ band).

The excited states belonging to $[\mathbf{5 6}, \ell]$ multiplets are rather simple and can be studied by analogy to the ground state. Naturally the splitting starts at order $1 / N_{c}$ $[11,14]$.

TABLE 1. List of operators and coefficients of the mass operator (1) for $\left[\mathbf{7 0}, 1^{-}\right]$.

\begin{tabular}{lrrrrrr}
\hline Operator & & Fit 1 (MeV) & Fit 2 (MeV) & Fit 3 (Mev) & Fit 4 (MeV) & Fit 5 (MeV) \\
\hline$O_{1}=N_{c} \mathbb{1 l}$ & $c_{1}=$ & $481 \pm 5$ & $482 \pm 5$ & $484 \pm 4$ & $484 \pm 4$ & $498 \pm 3$ \\
$O_{2}=\frac{1}{N_{c}} \ell^{i} S^{i}$ & $c_{2}=$ & $-47 \pm 39$ & $-30 \pm 34$ & $-31 \pm 20$ & $8 \pm 15$ & $38 \pm 34$ \\
$O_{3}=\frac{1}{N_{c}} S^{i} S^{i}$ & $c_{3}=$ & $161 \pm 16$ & $149 \pm 11$ & $159 \pm 16$ & $149 \pm 11$ & $156 \pm 16$ \\
$O_{4}=\frac{1}{N_{c}} T^{a} T^{a}$ & $c_{4}=$ & $169 \pm 36$ & $170 \pm 36$ & $138 \pm 27$ & $142 \pm 27$ & \\
$O_{5}=\frac{3}{N_{c}^{2}} \ell^{(2) i j} G^{i a} G^{j a}$ & $c_{5}=$ & $-443 \pm 459$ & & $-371 \pm 456$ & & $-514 \pm 458$ \\
$O_{6}=\frac{1}{N_{c}^{2}} \ell^{i} T^{a} G^{i a}$ & $c_{6}=$ & $473 \pm 355$ & $433 \pm 353$ & & & $-606 \pm 273$ \\
\hline$\chi_{\text {dof }}^{2}$ & & & & & 0.96 & 11.5 \\
\hline
\end{tabular}

The states belonging to $[\mathbf{7 0}, \ell]$-plets are more difficult due to the presence of a mixed symmetry. So far, in calculating the mass spectrum, the general practice was to split the baryon into an excited quark and a symmetric core, in this way reducing the problem to the well known ground state. There are two drawbacks in this procedure. One is that each generator of $\mathrm{SU}\left(2 N_{f}\right)$ is written as a sum of two terms, one acting on the excited quark and the other on the core. As a consequence, the number of linearly independent operators to be used in Eq. (2) increases tremendously and the number of coefficients to be determined becomes larger or much larger than the experimental data available. For example, for the $\left[\mathbf{7 0}, 1^{-}\right]$multiplet with $N_{f}=2$ one has 12 linearly independent operators up to order $1 / N_{c}$ [7], instead of 6 as in the present approach (see Table 1 ). We recall that there are only 7 nonstrange resonances belonging to this band. They are given in Table 2. Consequently, in selecting the most dominant operators one has to make an arbitrary choice [7]. The second drawback is due to the truncation of the wave function containing the orbital and the spin-flavor parts. The exact wave function is given by a linear combination of terms of equal weight where each term corresponds to a given Young tableau of mixed symmetry denoted by $\left[N_{c}-1,1\right]$. In the above procedure only the term where the last quark is in the second row was kept, the other $N_{c}-2$ terms being ignored. As a consequence the order of the spin-orbit operator became $N_{c}^{0}$. 
In this practice the matrix elements of the excited quark are straightforward, as being described by single-particle operators. The matrix elements of the core operators $S_{c}^{i}$ and $T_{c}^{a}$ are also simple to calculate, while $G_{c}^{i a}$ are more involved. Analytic group theoretical formulas for several types of matrix elements of the $\mathrm{SU}(4)$ generators have been derived in the late sixtieths [15], in the context of nuclear physics. Every matrix element is factorized according to a generalized Wigner-Eckart theorem into a reduced matrix element and an SU(4) ClebschGordan coefficient. Recently we have extended the approach to calculate isoscalar factors needed for the matrix elements of $\mathrm{SU}(6)$ generators between symmetric $\left[N_{c}\right]$ states [16].

The $\left[\mathbf{7 0}, \mathbf{1}^{-}\right]$-plet. Here we propose a new method where the splitting into an excited quark and a core is unnecessary. Details can be found in Ref. [17]. All one needs to know are the matrix elements of the $\mathrm{SU}\left(2 N_{f}\right)$ generators between mixed symmetric states $\left[N_{c}-1,1\right]$. For $N_{f}=2$ these are provided by the work of Hecht and Pang [15]. To our knowledge such matrix elements are yet unknown for $N_{f}=3$. Thus our work deals with nonstrange baryon resonances only. The list of operators $O_{i}$ contributing to the mass operator (1) is shown in Table 1 together with the coefficients $c_{i}$. The first column gives all linearly independent operators of type (2) up to order $1 / N_{c}$. The other columns indicate the values obtained for the dynamical coefficients $c_{i}$ from various fits. Fit 1 contains all operators and gives the best $\chi_{\text {dof }}^{2}$. In the other columns one or two operators have been removed in order to understand their role in the fit. One can see that the removal of $O_{5}$ or of $O_{6}$ is not so dramatic but that the removal of $O_{4}$ badly deteriorates the fit. The data are from Ref. [18]. It is important to note that here the angular momentum operator of components $\ell_{i}(i=1,2,3)$ is an intrinsic operator, acting on the entire system, not on the $N_{c}$-th quark only, as in previous studies, which means it was taken relative to a fixed center of mass [7]. In other words here we do not have a center of mass problem which may complicate the $N_{c}$ counting.

TABLE 2. Partial contributions and total mass $(\mathrm{MeV})$ of $\left[\mathbf{7 0}, \mathbf{1}^{-}\right]$resonances predicted by the $1 / N_{c}$ expansion. The last two columns reproduce the experimental masses and the status of resonances [18].

\begin{tabular}{|c|c|c|c|c|c|c|c|c|c|}
\hline & \multicolumn{6}{|c|}{ Part. contrib. (MeV) } & \multirow[t]{2}{*}{ Total $(\mathrm{MeV})$} & \multirow[t]{2}{*}{ Exp. $(\mathrm{MeV})$} & \multirow[t]{2}{*}{ Name, status } \\
\hline & $c_{1} O_{1}$ & $c_{2} \mathrm{O}_{2}$ & $c_{3} \mathrm{O}_{3}$ & $c_{4} \mathrm{O}_{4}$ & $c_{5} O_{5}$ & $c_{6} \mathrm{O}_{6}$ & & & \\
\hline${ }^{2} N_{\frac{1}{2}}$ & 1444 & -16 & 40 & 42 & 0 & -13 & $1529 \pm 11$ & $1538 \pm 18$ & $S_{11}(1535)^{* * * *}$ \\
\hline${ }^{4} N_{\frac{1}{2}}$ & 1444 & 39 & 201 & 42 & -31 & -33 & $1663 \pm 20$ & $1660 \pm 20$ & $S_{11}(1650)^{* * * *}$ \\
\hline${ }^{2} N_{\frac{3}{2}}$ & 1444 & -8 & 40 & 42 & 0 & 7 & $1525 \pm 8$ & $1523 \pm 8$ & $D_{13}(1520)^{* * * *}$ \\
\hline${ }^{4} N_{\frac{3}{2}}$ & 1444 & 16 & 201 & 42 & 25 & -13 & $1714 \pm 45$ & $1700 \pm 50$ & $D_{13}(1700)^{* * *}$ \\
\hline${ }^{4} N_{\frac{5}{2}}$ & 1444 & -24 & 201 & 42 & -6 & 20 & $1677 \pm 8$ & $1678 \pm 8$ & $D_{15}(1675)^{* * * *}$ \\
\hline${ }^{2} \Delta_{\frac{1}{2}}$ & 1444 & 16 & 40 & 211 & 0 & -66 & $1645 \pm 30$ & $1645 \pm 30$ & $S_{31}(1620) * * * *$ \\
\hline${ }^{2} \Delta_{\frac{3}{2}}$ & 1444 & -8 & 40 & 211 & 0 & -33 & $1720 \pm 50$ & $1720 \pm 50$ & $D_{33}(1700)^{* * * *}$ \\
\hline
\end{tabular}


The partial contributions and the total mass predicted by the $1 / N_{c}$ expansion are given in Table 2. One can see that the contributions of all terms containing angular momentum, and in particular that of the spin-orbit, are small. This can be viewed as a dynamical effect. An entirely new quantitative result is that the isospin-isospin term $\mathrm{O}_{4}$ brings a dominant contribution to $\Delta$ resonances, of the same order as the spin-spin term $\mathrm{O}_{3}$ brings to $N$ resonances.

Conclusions. These results shed a new light into the description of the baryon multiplet $\left[\mathbf{7 0}, 1^{-}\right]$in the $1 / N_{c}$ expansion. The main findings are:

- In the mass formula the expansion starts at order $1 / N_{c}$, as for the ground state, instead of $N_{c}^{0}$ as previously concluded.

- The isospin operator $O_{4}=\frac{1}{N_{c}} T^{a} T^{a}$ is crucial in the fit to the existing data and its contribution is as important as that of the spin term $O_{3}=\frac{1}{N_{c}} S^{i} S^{i}$.

It would be interesting to reconsider the study of higher excited baryons, for example those belonging to $\left[\mathbf{7 0}, \ell^{+}\right]$multiplets, in the spirit of the present approach. Based on group theoretical arguments it is expected that the mass splitting starts at order $1 / N_{c}$, as a general rule.

\section{REFERENCES}

1. G. 't Hooft, Nucl. Phys. 72 (1974) 461; E. Witten, Nucl. Phys. B160 (1979) 57.

2. J. L. Gervais and B. Sakita, Phys. Rev. Lett. 52 (1984) 87; Phys. Rev. D 30 (1984) 1795.

3. R. Dashen and A. V. Manohar, Phys. Lett. B315 (1993) 425; ibid B315 (1993) 438.

4. R. Dashen, E. Jenkins, and A. V. Manohar, Phys. Rev. D51 (1995) 3697.

5. E. Jenkins, Ann. Rev. Nucl. Part. Sci. 48 (1998) 81

6. J. L. Goity, Phys. Lett. B414 (1997) 140.

7. C. E. Carlson, C. D. Carone, J. L. Goity and R. F. Lebed, Phys. Rev. D59 (1999) 114008.

8. C. E. Carlson and C. D. Carone, Phys. Rev. D58 (1998) 053005.

9. J. L. Goity, C. L. Schat and N. N. Scoccola, Phys. Rev. D66 (2002) 114014.

10. C. E. Carlson and C. D. Carone, Phys. Lett. B484 (2000) 260.

11. J. L. Goity, C. L. Schat and N. N. Scoccola, Phys. Lett. B564 (2003) 83.

12. N. Matagne and Fl. Stancu, Phys. Lett B631 (2005) 7.

13. N. Matagne and Fl. Stancu, Phys. Rev. D74 (2006) 034014

14. N. Matagne and Fl. Stancu, Phys. Rev. D71 (2005) 014010.

15. K. T. Hecht and S. C. Pang, J. Math. Phys. 10 (1969) 1571.

16. N. Matagne and Fl. Stancu, Phys. Rev. D73 (2006) 114025

17. N. Matagne and Fl. Stancu, arXiv:hep-ph/0610099.

18. W.-M. Yao et al. [Particle Data Group], J. Phys. G33 (2006) 1. 